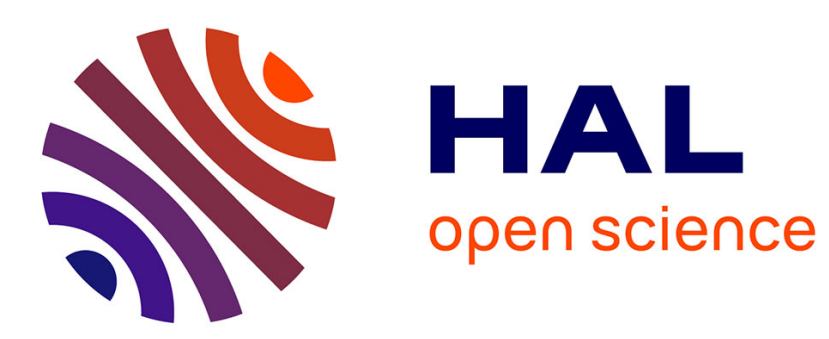

\title{
Can extinction rates be estimated without fossils?
}

Emmanuel Paradis

\section{To cite this version:}

Emmanuel Paradis. Can extinction rates be estimated without fossils?. Journal of Theoretical Biology, 2004, 229 (1), pp.19-30. 10.1016/j.jtbi.2004.02.018 . ird-02063037

\section{HAL Id: ird-02063037 https://hal.ird.fr/ird-02063037}

Submitted on 10 Mar 2019

HAL is a multi-disciplinary open access archive for the deposit and dissemination of scientific research documents, whether they are published or not. The documents may come from teaching and research institutions in France or abroad, or from public or private research centers.
L'archive ouverte pluridisciplinaire HAL, est destinée au dépôt et à la diffusion de documents scientifiques de niveau recherche, publiés ou non, émanant des établissements d'enseignement et de recherche français ou étrangers, des laboratoires publics ou privés. 


\title{
Can extinction rates be estimated without fossils?
}

\author{
Emmanuel Paradis* \\ Laboratoire de Paléontologie, Paléobiologie \& Phylogénie, \\ Institut des Sciences de l'Évolution, \\ Université Montpellier II, \\ F-34095 Montpellier cédex 05, France
}

*E-mail: paradis@isem.univ-montp2.fr; Tel: +33 4671439 64; fax: +33 467143610 


\begin{abstract}
There is considerable interest in the possibility of using molecular phylogenies to estimate extinction rates. The present study aims at assessing the statistical performance of the birth-death model fitting approach to estimate speciation and extinction rates by comparison to the approach considering fossil data. A simulation-based approach was used. The diversification of a large number of lineages was simulated under a wide range of speciation and extinction rate values. The estimators obtained with fossils performed better than those without fossils. In the absence of fossils (e.g., with a molecular phylogeny), the speciation rate was correctly estimated in a wide range of situations; the bias of the corresponding estimator was close to zero for the largest trees. However, this estimator was substantially biased when the simulated extinction rate was high. On the other hand the estimator of extinction rate was biased in a wide range of situations. Surprisingly, this bias was lesser with medium-sized trees. Some recommendations for interpreting results from a diversification analysis are given.
\end{abstract}

Keywords: Diversity; Estimation; Extinction; Maximum likelihood; Phylogeny; Simulation; Speciation 


\section{Introduction}

The tempo and mode of evolution has been one of the fundamental questions in evolutionary biology (Simpson, 1953). Recent advances in phylogenetics have given a fresh look at this issue (Barraclough and Nee, 2001). The reconstruction of the relationships among species allows one to test whether the shape of the reconstructed phylogeny agrees with a given model of diversification (e.g., Kirkpatrick and Slatkin, 1993; Slowinski and Guyer, 1993; McKenzie and Steel, 2000). In the situation where the branches of the phylogeny have estimated lengths, it is possible to fit models in order to estimate the rates of diversification of the studied lineage (Nee et al., 1994b; Paradis, 1997).

Conceptually, the diversification of a lineage may be seen as a series of speciation and extinction events through time. Speciation events give birth to new species, and extinction events result in the death of species. This representation is practical for modelling the diversification of lineages since, with some further assumptions, this agrees with the birth-and-death processes which have been extensively studied in the past (Kendall, 1948a,b, 1949; Darwin, 1956; Keiding, 1975).

Nee et al. (1994b) proposed a method to estimate both speciation and extinction rates of a lineage using the reconstructed phylogenetic relationships of the living species, for instance using molecular data. They developed a likelihood-based approach to estimate both speciation and extinction rates. It follows that the extinction rate of a lineage could be estimated even in the absence of fossils, and thus without observing any event of extinction (Nee et al., 1995). This conjecture may seem counterintuitive since the information on extinction comes from extinct species: for instance, when a phylogeny with fossils is analysed, the estimation of extinction rate is done with the ratio of the number of extinction events on the cumulative numbers of species. Clearly, the number of extinction events cannot be observed without fossils. 
Kubo and Iwasa (1995) developed a method close to Nee et al.'s (1994b): they used the same birth-death model but they fitted this model with a polytope algorithm instead of maximum likelihood. Kubo and Iwasa (1995) then showed, using simulations, that the estimator of extinction rates has a too large variance to be reliable.

It is important to assess the precision of the method introduced by Nee et al. (1994b) since it could have many potential applications in the study of organisms poorly represented in the fossil record, such as soft-bodied organisms. This method may be used also to study the dynamics of viral populations (Nee et al., 1995). The goal of the present study is to assess the statistical performance of this method in a wide range of parameter values. Nee et al.'s (1994b) method performance was compared to the performance of the method where fossils, and thus past extinction events, are observed.

\section{Methods}

A large number of phylogenies were simulated with different values of speciation and extinction probabilities. The approach adopted was to simulate the speciation and extinction events, rather than generate sets of branching times from theoretical distributions, in order to mimic as close as possible the evolutionary process. Simulations were started with a single species. At each time step, each species living in the lineage had a probability (denoted $\mu$ ) to die. If it survived, each species had then a probability (denoted $\lambda$ ) to generate two daughter-species, otherwise it simply survived to the next time step. This process was simulated during 1000 time steps; all speciation and extinction events were recorded.

The probability of speciation $\lambda$ varied between 0.0001 and 0.005 with a step of 0.0001 , and for each of these values, $\mu$ varied between 0 and $\lambda-0.0001$ with the same step of 0.0001 . The constraint $\lambda>\mu$ was imposed so that the risk of extinction of the entire lineage was minimized. For each combination of these two parameters, the 
simulation was replicated 1000 times. Thus, a total of 2,500,000 simulations were started (50 values of $\lambda \times 50$ values of $\mu \times 1000$ replicates). Since the simulations were fully stochastic, so were the number of species at any time step. The lineages with 0,1 , or 2 living species at the end of the simulation were discarded. For the other lineages, two phylogenies were recorded: the first one with all speciation and extinction events so that the extinct species are included, and the second one with only the species living at the end of the simulation.

The phylogenies with only the living species were analysed with Nee et al.'s (1994b) method. This method fits a birth-death process (Kendall, 1948b) to the set of branching times calculated from the phylogeny. A re-parametrization is needed to allow the model fitting so that the parameters under consideration are $r=\lambda-\mu$, and $a=\mu / \lambda$ (see Nee et al., 1994b). The model is then fitted by maximum likelihood to give the estimates of both parameters, denoted $\hat{r}$ and $\hat{a}$, respectively.

The phylogenies with the living and the extinct species were analysed with the method described by Keiding (1975) who gave the following two maximum likelihood estimators of the speciation and extinction rates:

$$
\hat{b}=\frac{B_{T}}{\int_{0}^{T} X_{t} \mathrm{~d} t}, \quad \hat{d}=\frac{D_{T}}{\int_{0}^{T} X_{t} \mathrm{~d} t},
$$

where $B_{T}$ is the number of speciation events between time 0 and $T, D_{T}$ is the number of extinction events during the same time, and $X_{t}$ is the number of species living at time $t$. The integral at the denominators of eqn (1) was calculated as the sum of the branch lengths of the phylogeny.

The analyses of the simulated data were all done with R (Ihaka and Gentleman, 1996; R Development Core Team, 2003) using a package specially developed for phylogenetic analyses (Paradis et al., 2004). For all simulated lineages, the estimates $\hat{r}, \hat{a}, \hat{b}$, and $\hat{d}$ were stored together with the number of tips in both phylogenies, and the depth of the 
phylogeny without fossils (age of the youngest common ancestor of all living species). Note that the depth of the phylogeny with fossils was always 1000 time steps.

The relative error of each estimated parameter was calculated using:

$$
\frac{\hat{r}-(\lambda-\mu)}{\lambda-\mu}, \quad \frac{\hat{a}-\mu / \lambda}{\mu / \lambda}, \quad \frac{\hat{b}-\lambda}{\lambda}, \quad \frac{\hat{d}-\mu}{\mu} .
$$

Using relative errors avoids having large errors due to the fact that the values of the parameter are large as well. In the simulations with $\mu=0$, the relative error of $\hat{a}$ could not be calculated and was replaced by its absolute error: $\hat{a}-\mu / \lambda$. On the other hand, when $\mu=0$ the relative error of $\hat{d}$ was not considered because there was no extinction event $\left(D_{T}=0\right)$, and thus $\hat{d}=0$ in all replications.

Giving the very large amount of simulated data, summary statistics of the results were computed. The variations in the relative errors of the estimated parameters were investigated with respect to four variables: the true values of $\lambda$ and $\mu$, the number of tips of the phylogenies, and its depth. To give a picture of how the mean tendency and the dispersion of the relative errors varied, the results were summarized using box-and-whiskers plots. The relative errors were first dispatched in different categories with respect to: (i) each value of $\lambda$, (ii) each value of $\mu$, (iii) an interval of the number of tips, or (iv) an interval of the depth of the tree. For the number of tips and the depth of the tree, the intervals were created with an algorithm in $\mathrm{R}$ in order to make categories with approximately similar numbers. The numbers of phylogenies analysed in each category are given in the Appendix.

In a second step, for each category a box-and-whiskers was drawn where the box represents the lower and upper quartiles (thus $50 \%$ of the data are within the box), the median being indicated by a horizontal line inside the box. The upper and lower limits of the whiskers extend up to the quantiles $Q_{0.05}$ and $Q_{0.95}$, respectively (so that $90 \%$ of the data are between the limits of the whiskers. Giving that a few points in these plots are 
extreme outliers, particularly towards the positive values (data not shown), showing the extreme of these distributions with the whiskers (as usually done with such plots, Venables and Ripley 2002) would increase the range of the $y$-axis by several orders of magnitude, and would result in tiny unmeaningful boxes. These summary statistics were computed for each category of $\lambda$, of $\mu$, of the phylogeny depth, and of the number of tips of the phylogeny without fossils for the relative error of $\hat{r}$ and the absolute error of $\hat{a}$. They were also computed for each category of $\lambda$, of $\mu$, and of the number of tips of the phylogeny with fossils for the relative errors of $\hat{b}$ and of $\hat{d}$.

To allow a more direct comparison of both kinds of estimators, estimates of the speciation and extinction rates without fossils (denoted $\hat{b}_{o}$ and $\hat{d}_{o}$, respectively) were computed by back transformation of $\hat{r}$ and $\hat{a}$ :

$$
\hat{d}_{o}=\frac{\hat{r} \hat{a}}{1-\hat{a}}, \quad \hat{b_{o}}=\hat{r}+\hat{d}_{o}
$$

The relative errors of $\hat{b}_{o}$ and $\hat{d}_{o}$ were calculated in the same way than for $\hat{b}$ and $\hat{d}$.

To give a global picture of the bias in $\hat{b}_{o}$ and $\hat{d}_{o}$, the relative errors of these estimates were plotted with respect to the number of tips of the analysed tree for different values of $\lambda$ and of $\mu$. Five values of $\lambda$ were selected $(0.003,0.0035,0.004,0.0045$, and 0.005$)$, and six values of $\mu(0,0.0009,0.0014,0.0019,0.0024$, and 0.0029$)$ for this analysis. A local polynomial fit (Venables and Ripley, 2002, p. 230) was performed on each plot.

\section{Results}

The 2,500,000 simulations yielded 604,165 lineages that were amenable to further analyses (that is they had at least three species living after 1000 times steps). Fig. 1 shows an example of such a simulated lineage with both derived phylogenies. Among the 604,165 phylogenies considering only living species, estimation of $r$ and $a$ was not possible in 112,083 cases (there was no convergence of the fitting algorithm). This 
failure was clearly related to the number of tips in the tree: the mean number of tips among these 112,083 trees was $5.73(\mathrm{sd}=2.94$, median $=5$, maximum $=32)$, whereas it was $31.63(\mathrm{sd}=46.67$, median $=16$, maximum $=1140)$ among the 492,082 other trees.

The number of tips varied between 3 and 1140 for the phylogenies without fossils, and between 3 and 1161 for the phylogenies with fossils. In both cases, the distribution had its maximum at the smallest value, and was highly skewed towards the largest ones.

Fig. 2 shows the results for $\hat{r}$. There was a positive bias in this estimator for the smallest values of $\lambda$, and the median of the relative error of $\hat{r}$ converged to zero for increasing values of $\lambda$ to be close to zero at $\lambda \approx 0.002$. The dispersion of the relative error of $\hat{r}$ also decreased with increasing values of $\lambda$. The opposite results were observed with respect to the values of $\mu$ : the median of the relative error of $\hat{r}$ was very large for the largest values of $\mu$ with a median of about 15 for $\mu=0.0049$. The median of the relative error of $\hat{r}$ was somewhat unsensitive to the number of tips with just a small negative bias for the phylogenies with three tips. However, the dispersion of this error was greatly influenced by the number of tips: this dispersion decreased continuously for all categories of number of tips. A positive bias in $\hat{r}$ was observed when the depth of the tree was less than about 400 time units. This bias progressively decreased to zero when the depth of the tree increased. The dispersion of relative error of $\hat{r}$ also decreased when the depth of the tree increased to stabilize at a depth of about 700 time units.

Fig. 3 shows the results for $\hat{a}$. With respect to $\lambda$, the relative error of $\hat{a}$ was large for the small values of $\lambda$ and slowly converged to zero when $\lambda$ increased. The relative error of $\hat{a}$ was greatly affected by the value of $\mu$ : there was a systematic negative bias in $\hat{a}$ meaning that it underestimated the actual value of $a$ in most situations. On the other hand, the dispersion of the relative error of $\hat{a}$ decreased greatly with increasing values of $\mu$. The effect of the number of tips of the tree on $\hat{a}$ was complex: there was a negative bias in $\hat{a}$ for the smallest numbers of tips, and this bias converged to zero with increasing 
numbers. The median of the relative error of $\hat{a}$ was 0 for trees with 16 tips, but a negative bias was observed for trees with more tips. With respect to tree depth, the median relative error of $\hat{a}$ converged progressively to zero with increasing values of tree depth. The dispersion of the relative error of $\hat{a}$ was large for all values of tree depth.

Fig. 4 shows the results for $\hat{b}$. The relative error of $\hat{b}$ was very large for very small values of $\lambda$, and its median quickly converged to zero with increasing $\lambda$. It is remarkable that the effect of $\lambda$ on $\hat{b}$ was very similar to that on $\hat{r}$ with two differences, however: the dispersion of the relative error of $\hat{r}$ was greater (for $\lambda=0.005$, the first and third quartiles were $-0.118,0.067$ for $\hat{b}$, and $-0.264,0.160$ for $\hat{r}$ ), and the median of the relative error of $\hat{b}$ converged to zero more quickly though the difference was slight but systematic (for $\lambda=0.0016$, the median relative errors of $\hat{b}$ and $\hat{r}$ were 0.099 and 0.203 , respectively). The relative error in $\hat{b}$ was only slightly affected by the value of the extinction rate with a small positive bias for the large values of $\mu$. In all cases, the dispersion of $\hat{b}$ was small with $50 \%$ of the values being between -0.2 and 0.2 . As previously for $\hat{r}$ and $\hat{a}$, a negative bias in $\hat{b}$ for the smallest numbers of tips was observed. However, the dispersion of the relative error of $\hat{b}$ was much smaller than for $\hat{r}$.

Fig. 5 shows the results for $\hat{d}$. The relative error of $\hat{d}$ was greatly influenced by $\lambda$ with a strong negative bias for the smallest values of speciation rate and a progressive convergence of the median with increasing values. With respect to $\mu$, there was a strong negative bias for the smallest values of extinction rate with a quick convergence to zero with increasing $\mu$. However, a negative bias was observed for the largest values of $\mu$. The dispersion of the relative error in $\hat{d}$ continuously decreased with increasing values of $\mu$. As for the other estimators, the relative error in $\hat{d}$ was affected by the number of tips in the tree, but the dispersion was greater than for $\hat{b}$.

The estimates of speciation rate with $(\hat{b})$ and without $\left(\hat{b_{o}}\right)$ fossils were compared for the different values of $\lambda$ and $\mu$ (Fig. 6). With respect to $\lambda$, the performance of both 
estimators were very close except for the very small values of $\lambda$ where the estimates with fossils were somehow better than without, though a positive bias was also observed for the former. In terms of dispersion (as measured by the inter-quartile range), the estimates with fossils were slightly better than without fossils, particularly for the largest values of $\lambda$. With respect to $\mu$, the performances were more contrasted. With fossils, the bias in $\hat{b}$ was close to zero for all values of $\mu$ but showed a positive trend with increasing values of $\mu$. Without fossils, the bias in $\hat{b}_{o}$ showed a negative trend, and was much stronger than for $\hat{b}$ for the largest values of $\mu$. The contrast was even stronger when considering the dispersion of the estimates: the dispersion of $\hat{b_{o}}$ was always stronger than the dispersion of $\hat{b}$ and increased with increasing values of $\mu$, whereas the dispersion of $\hat{b_{o}}$ decreased with increasing values of $\mu$.

The same comparison was done between $\hat{d}$ and $\hat{d}_{o}$ (Fig. 6). With respect to $\lambda$, there was always a negative bias for both estimators, and they both converged to zero with increasing values of $\lambda$ but the convergence was much quicker for $\hat{d}$ than for $\hat{d}_{o}$. The dispersion of these estimates showed opposite trends, though the results were somewhat more complicated. With fossils, the dispersion of $\hat{d}$ increased with increasing values of $\lambda$, but then decreased for $\lambda>0.0023$. On the other hand, the dispersion of $\hat{d}_{o}$ increased for all values of $\lambda$, though it reached a plateau for $0.0003<\lambda<0.0023$. With respect to $\mu$, both estimators showed parallel results but the performances of $\hat{d}$ were much better than for $\hat{d}_{o}$, in terms of bias (the median of the relative error of $\hat{d}$ was much closer to zero than the one of $\hat{d}_{o}$ ) as well as in terms of dispersion of the estimates.

The analysis of the relative error of $\hat{b_{0}}$ with respect to the number of tips showed very similar results for the different values of $\lambda$ and $\mu$ (Fig. 7). In all cases the fitted curve was close to zero for a number of tips between 10 and 20 or more. The remarkable difference among the different plots was the maximum number of tips which was clearly related to the difference $\lambda-\mu$. All plots also showed similar results for the dispersion of the 
relative error of the estimates with a range of values quite similar, and a decrease in dispersion with an increasing number of tips.

The same analysis for $\hat{d}_{o}$ showed more complex results (Fig. 8). When $\mu=0$ the dispersion of the relative error of $\hat{d}_{o}$ (as shown by the range of the $y$-axes) was very low, and the fitted curves converged to zero with growing numbers of tips. On the other hand, when $\mu>0$ the fitted curves were almost always negative, increased with growing numbers of tips up to 10-20 tips, and then decreased afterwards. There was a continuous variation though from the smallest to the largest values of $\mu$ : positive values of the fitted curves were observed for $\mu=0.0009$, whereas the fitted curves were well below zero for $\mu=0.0029$. The dispersion of the relative error of $\hat{d}_{o}$ decreased with increasing values of both $\lambda$ and $\mu$.

\section{Discussion}

There is undoubtedly considerable interest in the possibility to use molecular phylogenies (or any phylogeny inferred from extant species) to estimate extinction rates since this approach does not require to collect data through time. However, it is necessary to assess the biases and limits of this approach which is the goal of this paper. The assessment of the bias of the estimators with fossils was done mainly for comparison: since the lineages that went extinct before 1000 time steps were not considered, the biases of these estimators were not correctly estimated.

Overall, the estimators obtained with fossils $(\hat{b}$ and $\hat{d})$ performed better than those without fossils ( $\hat{r}$ and $\hat{a})$ : the former had generally smaller bias and smaller variance than the former. All four estimators performed better with increasing values of speciation rate which is obviously due to the fact that high values of $\lambda$ result in more speciation events in the simulated trees eihter with or without fossils. On the other hand, when the extinction rate was high, all estimators were biased since most trees went extinct and 
thus only those with a low realized value of $\mu$ were effectively analysed.

All estimators behaved generally well with respect to the number of tips: the median errors were close to zero for 15 or more tips. However, the dispersion of all four estimators was continuously influenced by the number of tips: it was lowest for the largest number of tips. An exception to this pattern was $\hat{a}$ which showed a negative bias for the lowest and highest numbers of tips. Clearly, more tips result in more data to analyse, and thus it is expected that the estimators are more accurate (median close to zero, and low dispersion). In the case of $\hat{a}$, the negative bias for the highest numbers of tips may be due to the fact that most large trees were simulated with a low value of $\mu$ and a high value of $\lambda$. Consequently, these trees had a very low ratio $\lambda / \mu(=a)$ which critically affected the relative error of $\hat{a}$. It is noteworthy that this negative bias for the highest numbers of tips was also observed when considering the absolute error of $\hat{a}$, but was much slighter than for the relative error (results not shown).

In the trees without fossils, the most recent common ancestor to all living species (i.e. the root of the tree) varied randomly depending on the simulation. The median relative error of $\hat{r}$ was close to zero for tree depth values of about 450 or more, whereas a value of about 900 or more was required to obtain a median error of $\hat{a}$ close to zero. The depth of a tree is clearly related to extinction rate: the highest the value of $\mu$, the lowest the probability of a species appearing early in the simulation to survive until present.

It should be noted that all the results in this study were obtained with trees, either with or without fossils, which were known without error in terms of unlabelled topology and branch lengths. This is unlikely to be always true in real situations since there are many sources of error in estimating phylogenetic trees as clearly illustrated by various phylogenetic studies (see Whelan et al., 2001, for a review). If these errors in estimating trees are uniformly distributed along the tree, it should be expected that the median errors of the estimators studied here are not affected, though their dispersion are likely to 
be increased since a supplementary source of variation is added. On the other hand, if a systematic bias in estimating the trees exists, this will add a bias in the estimators of diversification.

Another assumption of the present study was that all species of the lineage are included in the reconstructed phylogenies. This is likely to be untrue in real situations. With fossils, many species are likely to have not been fossilized and thus cannot be included in a possible phylogenetic study. Without fossils, it is rare to have all living species of a clade to be included in a phylogenetic reconstruction. In the context of testing for temporal variation in diversification, it was shown that missing taxa in phylogenies induce a bias resulting in a substantial increase in the type I error rate (Nee et al., 1994a; Pybus and Harvey, 2000). There has been no assessment of the possible bias of the estimators $\hat{r}$ and $\hat{a}$ when a phylogeny is incomplete. However, it could be speculated from the present study that missing taxa may have an effect on the estimation of $r$ and $a$. Removing randomly some species from a clade can be seen as similar to analysing a smaller clade with the same parameters $\lambda$ and $\mu$. Thus the effects of missing species can be predicted from the effects of the number of tips observed on Figs. 7 and 8. Consequently, missing taxa in phylogenies are likely to bring about a negative bias in $\hat{r}$ (and $\hat{b_{o}}$ ), whereas the effect on $\hat{a}$ (and $\hat{d}_{o}$ ) would be more complex. In both cases, the bias is likely to be slight if the number of missing species is low.

It appears that accurate estimation of both speciation and extinction rates can be achieved only with fossil data. In the absence of fossils (e.g., with a molecular phylogeny), only the difference between these rates $(r)$ can be estimated with some accuracy in a wide range of situations, notably when the speciation rate is relatively large compared to the extinction rate. On the other hand, the estimation of $a$ was inaccurate in a wide range of situation. This result was anticipated by Darwin (1956, p. 30) who stated that "If $N_{1}, \ldots, N_{k}$ [the cumulative numbers of species] are the only observed quantities, 
estimation of $\mu / \lambda$ is likely to be very inaccurate since the range of values of $\mu / \lambda$ giving the same set $N_{1}, \ldots, N_{k}$ with a reasonable probability is very large." The present study gives some empirical numerical support to Darwin's logical argument. Remarkably, accurate estimation of $a$ was achieved when the extinction rate was close to zero and the tree depth was close to the actual age of the lineage, suggesting that $a$ may be correctly estimated only when the tree without fossils is close to the tree with fossils (i.e. the 'real' tree of the lineage).

An unexpected result comes from the fact that the error in $\hat{d}_{o}$ increases with increasing number of tips in the tree (Figs. 7-8). By contrats to $\hat{a}$, this cannot be explained by the fact that larger trees are produced by larger values of $\lambda$ (see above). It seems rather that large trees produced with a moderate value of $\mu$ do not show a typical distribution of their branch lengths. This is further evidenced by the fact that trees with a moderate number of tips $(\approx 20)$ gave good results in terms of relative error of $\hat{d}_{o}$. Using the formula for the expected mean number of species in a clade after a time $T, e^{(\lambda-\mu) T}$ (Kendall, 1948b), it can be found that a clade with an expectation of 20 species after $T=1000$ is characterized by $\lambda-\mu \approx 0.003$.

\section{Conclusions and Recommendations}

The results from the present study can be used to define recommendations when interpreting an analysis of diversification. It is generally not possible to choose between both situations considered here, with or without fossils. Lineages with fossil data are often extinct, and those which are studied with a molecular phylogenetic approach usually have no or a poor fossil record. Indeed, the comparison between both kinds of estimators was not intended to define guidelines but to give a comparison for the estimators without fossils.

Phylogenies should be analysed as complete as possible: missing species are likely to 
introduce a negative bias in the estimation of $\lambda$, and an undetermined bias for $\mu$. A clade with at least 15 species is appropriate to estimate $\lambda$. This parameter is likely to be underestimated with smaller trees. Surprinsingly, $\mu$ is likely to be well estimated with medium-sized trees (with 10-20 species). However, $\mu$ is likely to be underestimated in most situations except if $\mu \approx 0$ where an overestimation is expected. In the latter case, the bias will be very small though. In all cases, it seems better to consider $\hat{d}_{o}$ as a lower bound of $\mu$.

If some informations are available on the age of the studied clade and the age of the most recent common ancestor of the species included in the phylogeny (called tree depth in the present paper), this may be used in interpreting the estimates of speciation and extinction rates. If the ratio of the latter on the former is less than 0.5 , then the estimate of $\lambda$ is likely to be positively biased. If this ratio is less than 0.9 , then the estimate of $\mu$ is likely to be negatively biased.

The present study is the first extensive analysis of the statistical performance of the birth-death estimators as applied to phylogenetic data. Nee (2001) used simulations to compare the statistical properties of several estimators but he considered only the birth-only model (also called Yule model). Some further studies are clearly needed, particularly to assess the robustness of the birth-death estimators when rates vary through time or across lineages since such situations are likely to be more biologically plausible than the homogeneous rates case considered here.

\section{Acknowledgements}

I am grateful to two anonymous referees for their constructive comments on a previous of this paper. This research was financially supported by the Institut Français de la Biodiversité and the Centre National de la Recherche Scientifique. This is publication 2004-009 of the Institut des Sciences de l'Évolution (Unité Mixte de Recherche 5554 du 
Centre National de la Recherche Scientifique).

\section{Appendix}

$\mathrm{T}$ able A1. Number of simulated lineages with respect to each value of speciation rate $\lambda$.

\begin{tabular}{lrlr}
\hline$\lambda$ & Number of trees & $\lambda$ & Number of trees \\
\hline 0.0001 & 10 & 0.0026 & 12094 \\
0.0002 & 52 & 0.0027 & 12717 \\
0.0003 & 192 & 0.0028 & 13245 \\
0.0004 & 339 & 0.0029 & 13883 \\
0.0005 & 639 & 0.003 & 14553 \\
0.0006 & 934 & 0.0031 & 15066 \\
0.0007 & 1277 & 0.0032 & 15729 \\
0.0008 & 1690 & 0.0033 & 16242 \\
0.0009 & 2054 & 0.0034 & 17044 \\
0.001 & 2648 & 0.0035 & 17528 \\
0.0011 & 3061 & 0.0036 & 18165 \\
0.0012 & 3602 & 0.0037 & 18581 \\
0.0013 & 4261 & 0.0038 & 19304 \\
0.0014 & 4755 & 0.0039 & 19701 \\
0.0015 & 5346 & 0.004 & 20505 \\
0.0016 & 5998 & 0.0041 & 21036 \\
0.0017 & 6526 & 0.0042 & 21535 \\
0.0018 & 7056 & 0.0043 & 21881 \\
0.0019 & 7707 & 0.0044 & 22362 \\
0.002 & 8331 & 0.0045 & 32434 \\
0.0021 & 8886 & 0.0046 & 23821 \\
0.0022 & 9691 & 0.0047 & 24213 \\
0.0023 & 10194 & 0.0048 & 24312 \\
0.0024 & 10849 & 0.0049 & 24990 \\
0.0025 & 11513 & 0.005 & 25613 \\
\hline
\end{tabular}


Table A2. Number of simulated lineages with respect to each value of extinction rate $\mu$.

\begin{tabular}{lrlr}
\hline$\mu$ & Number of trees & $\mu$ & Number of trees \\
\hline 0 & 37360 & 0.0025 & 8196 \\
0.0001 & 35528 & 0.0026 & 7571 \\
0.0002 & 34013 & 0.0027 & 6840 \\
0.0003 & 32422 & 0.0028 & 6448 \\
0.0004 & 30931 & 0.0029 & 6006 \\
0.0005 & 29406 & 0.003 & 5446 \\
0.0006 & 27971 & 0.0031 & 4845 \\
0.0007 & 26425 & 0.0032 & 4587 \\
0.0008 & 25073 & 0.0033 & 4162 \\
0.0009 & 23634 & 0.0034 & 3727 \\
0.001 & 22234 & 0.0035 & 3346 \\
0.0011 & 20566 & 0.0036 & 3040 \\
0.0012 & 19411 & 0.0037 & 2625 \\
0.0013 & 18449 & 0.0038 & 2461 \\
0.0014 & 17213 & 0.0039 & 2109 \\
0.0015 & 16106 & 0.004 & 1796 \\
0.0016 & 15516 & 0.0041 & 1571 \\
0.0017 & 14419 & 0.0042 & 1342 \\
0.0018 & 13450 & 0.0043 & 1117 \\
0.0019 & 12629 & 0.0044 & 918 \\
0.002 & 11751 & 0.0045 & 762 \\
0.0021 & 10774 & 0.0046 & 542 \\
0.0022 & 10137 & 0.0047 & 382 \\
0.0023 & 9492 & 0.0048 & 266 \\
0.0024 & 9030 & 0.0049 & 120 \\
\hline & & &
\end{tabular}


Table A3. Intervals defined to make groups with respect to the depth of the tree for the phylogenies without fossils.

\begin{tabular}{|c|c|c|c|}
\hline Depth of the tree & Number of trees & Depth of the tree & Number of trees \\
\hline $2-148$ & 9725 & $783-794$ & 9745 \\
\hline $149-220$ & 9849 & $795-806$ & 10010 \\
\hline $221-277$ & 9950 & $807-817$ & 9636 \\
\hline $278-324$ & 9751 & $818-828$ & 10059 \\
\hline $325-367$ & 9907 & $829-838$ & 9319 \\
\hline $368-404$ & 9751 & 839-848 & 9766 \\
\hline $405-438$ & 9912 & $849-858$ & 9931 \\
\hline $439-469$ & 9882 & $859-868$ & 10358 \\
\hline $470-498$ & 9783 & $869-877$ & 9322 \\
\hline $499-524$ & 9700 & $878-886$ & 9544 \\
\hline $525-549$ & 9963 & $887-895$ & 10048 \\
\hline $550-571$ & 9639 & 896-904 & 10072 \\
\hline $572-592$ & 9679 & $905-912$ & 9432 \\
\hline $593-612$ & 9779 & $913-921$ & 10816 \\
\hline $613-632$ & 10067 & $922-929$ & 9754 \\
\hline $633-650$ & 9757 & $930-936$ & 8740 \\
\hline $651-668$ & 10159 & $937-944$ & 10378 \\
\hline $669-684$ & 9564 & $945-952$ & 10558 \\
\hline $685-700$ & 9999 & $953-959$ & 9557 \\
\hline $701-715$ & 9724 & $960-966$ & 9814 \\
\hline $716-729$ & 9484 & $967-973$ & 9670 \\
\hline $730-744$ & 10433 & $974-980$ & 10065 \\
\hline $745-757$ & 9531 & $981-986$ & 8786 \\
\hline $758-770$ & 10090 & 987-993 & 10436 \\
\hline $771-782$ & 9568 & 994-1000 & 10620 \\
\hline
\end{tabular}


Table A4. Intervals defined to make groups with respect to the number of tips for the phylogenies without fossils.

\begin{tabular}{cr}
\hline Number of tips & Number of trees \\
\hline 3 & 59722 \\
4 & 48139 \\
5 & 40003 \\
6 & 33835 \\
7 & 29442 \\
8 & 25660 \\
9 & 22400 \\
10 & 19911 \\
11 & 17906 \\
12 & 16161 \\
13 & 14633 \\
14 & 13302 \\
15 & 12216 \\
16 & 11239 \\
$17-18$ & 20104 \\
19 & 8789 \\
$20-21$ & 16094 \\
22 & 7146 \\
$23-24$ & 13327 \\
$25-26$ & 11832 \\
$27-29$ & 15463 \\
$30-32$ & 13040 \\
$33-35$ & 11353 \\
$36-39$ & 12918 \\
$40-43$ & 11001 \\
$44-49$ & 13620 \\
$50-55$ & 11029 \\
$56-64$ & 13048 \\
$65-75$ & 12049 \\
$76-90$ & 11885 \\
$91-114$ & 12367 \\
$115-161$ & 12315 \\
$162-1140$ & 12216 \\
\hline & \\
\hline &
\end{tabular}


Table A5. Intervals defined to make groups with respect to the number of tips for the phylogenies with fossils.

\begin{tabular}{crcr}
\hline Number of tips & Number of trees & Number of tips & Number of trees \\
\hline 3 & 19538 & $24-25$ & 16913 \\
4 & 24095 & 26 & 7670 \\
5 & 25499 & $27-28$ & 14293 \\
6 & 25061 & $29-30$ & 12877 \\
7 & 24074 & $31-32$ & 11635 \\
8 & 23054 & $33-34$ & 10696 \\
9 & 21616 & $35-37$ & 14303 \\
10 & 20508 & $38-40$ & 12640 \\
11 & 19086 & $41-43$ & 11157 \\
12 & 18043 & $44-47$ & 13336 \\
13 & 16724 & $48-51$ & 11397 \\
14 & 15725 & $52-56$ & 12161 \\
15 & 14767 & $57-62$ & 12340 \\
16 & 13505 & $63-70$ & 13300 \\
17 & 12804 & $71-79$ & 11831 \\
18 & 12076 & $80-91$ & 12212 \\
19 & 11457 & $92-107$ & 11679 \\
20 & 10518 & $108-133$ & 12472 \\
$21-22$ & 19609 & $134-181$ & 12108 \\
23 & 9154 & $182-1166$ & 12232 \\
\hline
\end{tabular}

\section{References}

Barraclough, T. G., Nee, S., 2001. Phylogenetics and speciation. Trends Ecol. Evol. $16(7), 391-399$.

Darwin, J. H., 1956. The behaviour of an estimator for a simple birth and death process. Biometrika 43, 23-31.

Ihaka, R., Gentleman, R., 1996. R: a language for data analysis and graphics. J. Comput. Graph. Statist. 5 (3), 299-314.

Keiding, N., 1975. Maximum likelihood estimation in the birth-and-death process. Ann. Stat. 3 (2), 363-372. 
Kendall, D. G., 1948a. On some modes of population growth leading to R. A. Fisher's logarithmic series distribution. Biometrika 35 (1), 6-15.

Kendall, D. G., 1948b. On the generalized "birth-and-death" process. Ann. Math. Stat. $19,1-15$.

Kendall, D. G., 1949. Stochastic processes and population growth. J. R. Statist. Soc. B $11(2), 230-264$.

Kirkpatrick, M., Slatkin, M., 1993. Searching for evolutionary patterns in the shape of a phylogenetic tree. Evolution 47 (4), 1171-1181.

Kubo, T., Iwasa, Y., 1995. Inferring the rates of branching and extinction from molecular phylogenies. Evolution 49 (4), 694-704.

McKenzie, A., Steel, M., 2000. Distributions of cherries for two models of trees. Math. Biosci. 164, 81-92.

Nee, S., 2001. Inferring speciation rates from phylogenies. Evolution 55 (4), 661-668.

Nee, S., Holmes, E. C., May, R. M., Harvey, P. H., 1994a. Extinction rates can be estimated from molecular phylogenies. Phil. Trans. R. Soc. Lond. B 344, 77-82.

Nee, S., Holmes, E. C., Rambaut, A., Harvey, P. H., 1995. Inferring population history from molecular phylogenies. Phil. Trans. R. Soc. Lond. B 349 (1327), 25-31.

Nee, S., May, R. M., Harvey, P. H., 1994b. The reconstructed evolutionary process. Phil. Trans. R. Soc. Lond. B 344, 305-311.

Paradis, E., 1997. Assessing temporal variations in diversification rates from phylogenies: estimation and hypothesis testing. Proc. R. Soc. Lond. B 264, $1141-1147$.

Paradis, E., Claude, J., Strimmer, K., 2004. APE: analyses of phylogenetics and evolution in R language. Bioinformatics 20 (2), 289-290.

Pybus, O. G., Harvey, P. H., 2000. Testing macro-evolutionary models using incomplete molecular phylogenies. Proc. R. Soc. Lond. B 267, 2267-2272. 
R Development Core Team, 2003. R: a language and environment for statistical computing. R Foundation for Statistical Computing, Vienna, Austria, iSBN 3-900051-00-3.

URL http: / /www.R-project.org

Simpson, G. G., 1953. The major features of evolution. Columbia University Press, New York.

Slowinski, J. B., Guyer, C., 1993. Testing whether certain traits have caused amplified diversification: an improved method based on a model of random speciation and extinction. Am. Nat. 142 (6), 1019-1024.

Venables, W. N., Ripley, B. D., 2002. Modern applied statistics with S (fourth edition). Springer, New York.

Whelan, S., Liò, P., Goldman, N., 2001. Molecular phylogenetics: state-of-the-art methods for looking into the past. Trends Genet. 17 (5), 262-272. 


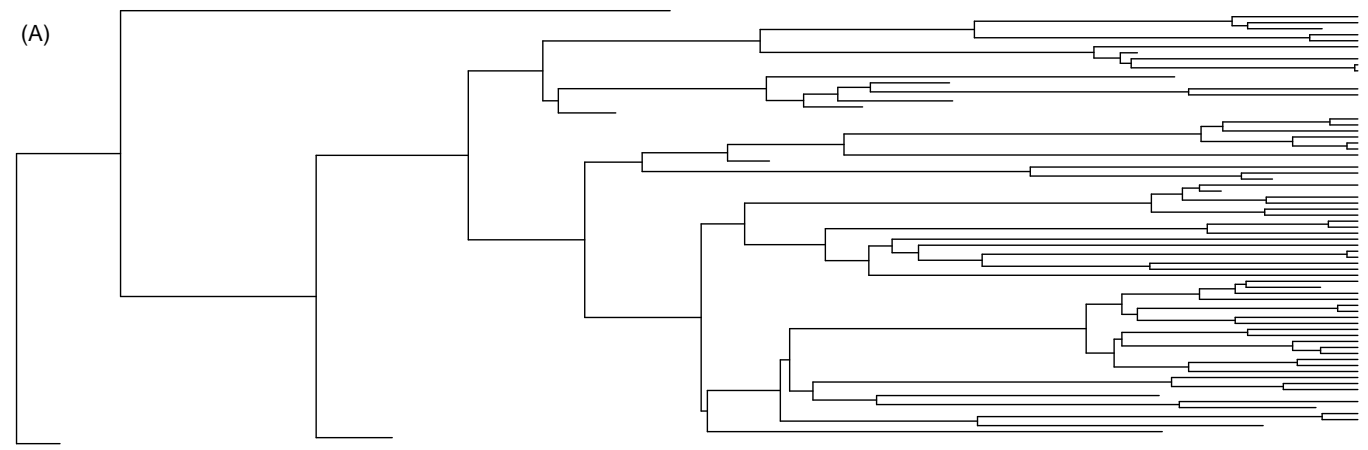

(B)

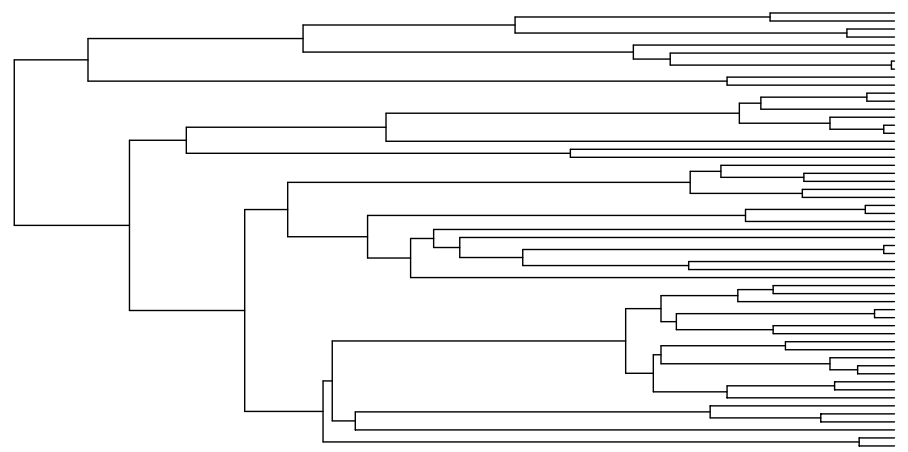

Figure 1: Illustration of the two phylogenies derived from the simulation of a lineage with speciation rate $\lambda=0.005$ and extinction rate $\mu=0.001$. (A) Complete phylogeny with the extinct species. (B) Phylogeny with only the species living at the end of the simulation. Note that the three most ancient species have disappeared from the data in (B). The parameter estimates for these data are: (A) $\hat{b}=0.0058, \hat{d}=0.0015$, and (B) $\hat{r}=0.0052, \hat{a}=0$. 

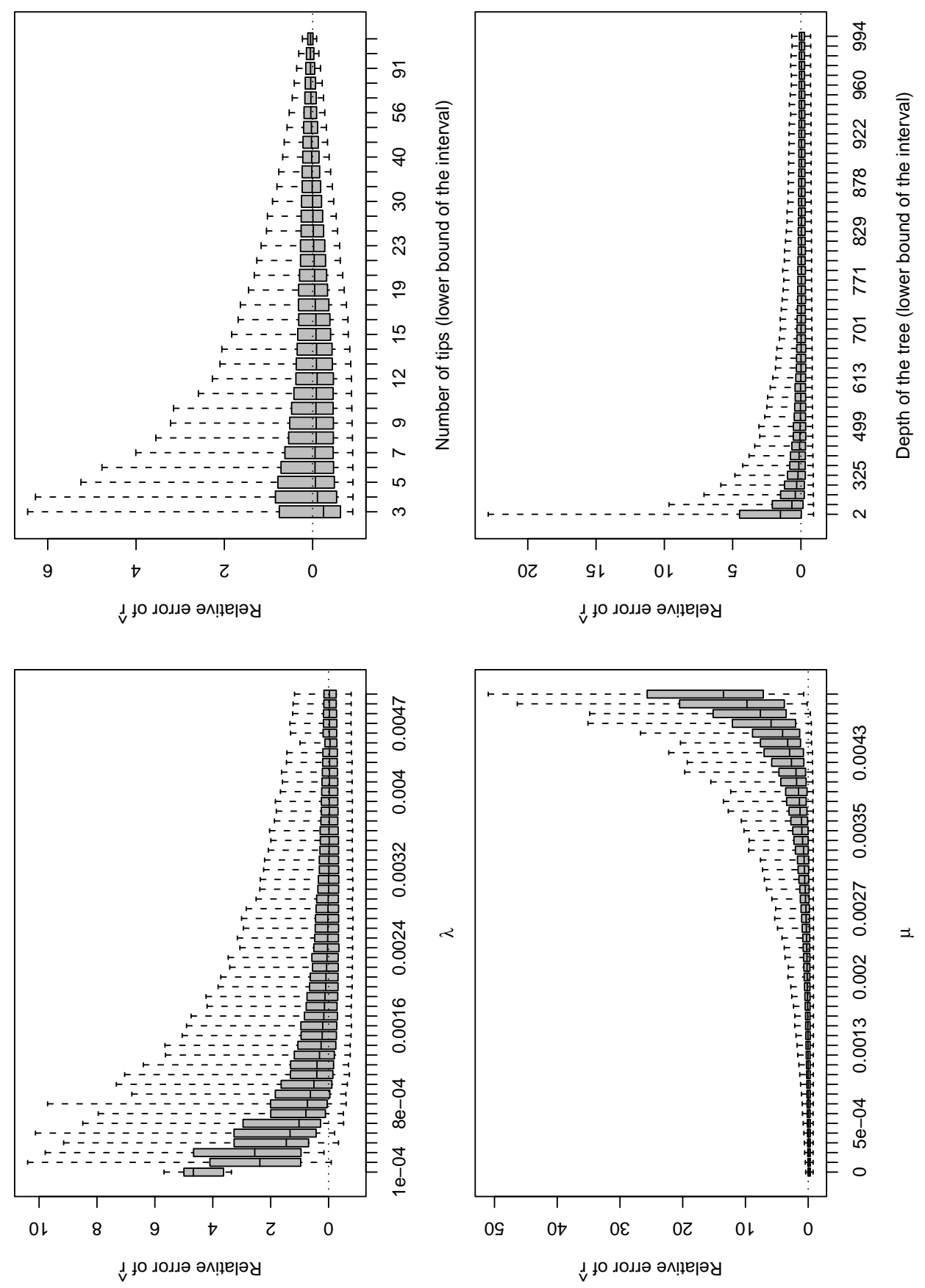

Figure 2: Results for $\hat{r}$. On each plot, the effect is considered alone and the trees are combined for all the different values of the other effects (e.g., the trees with the same value of $\lambda$ may have different number of tips). 

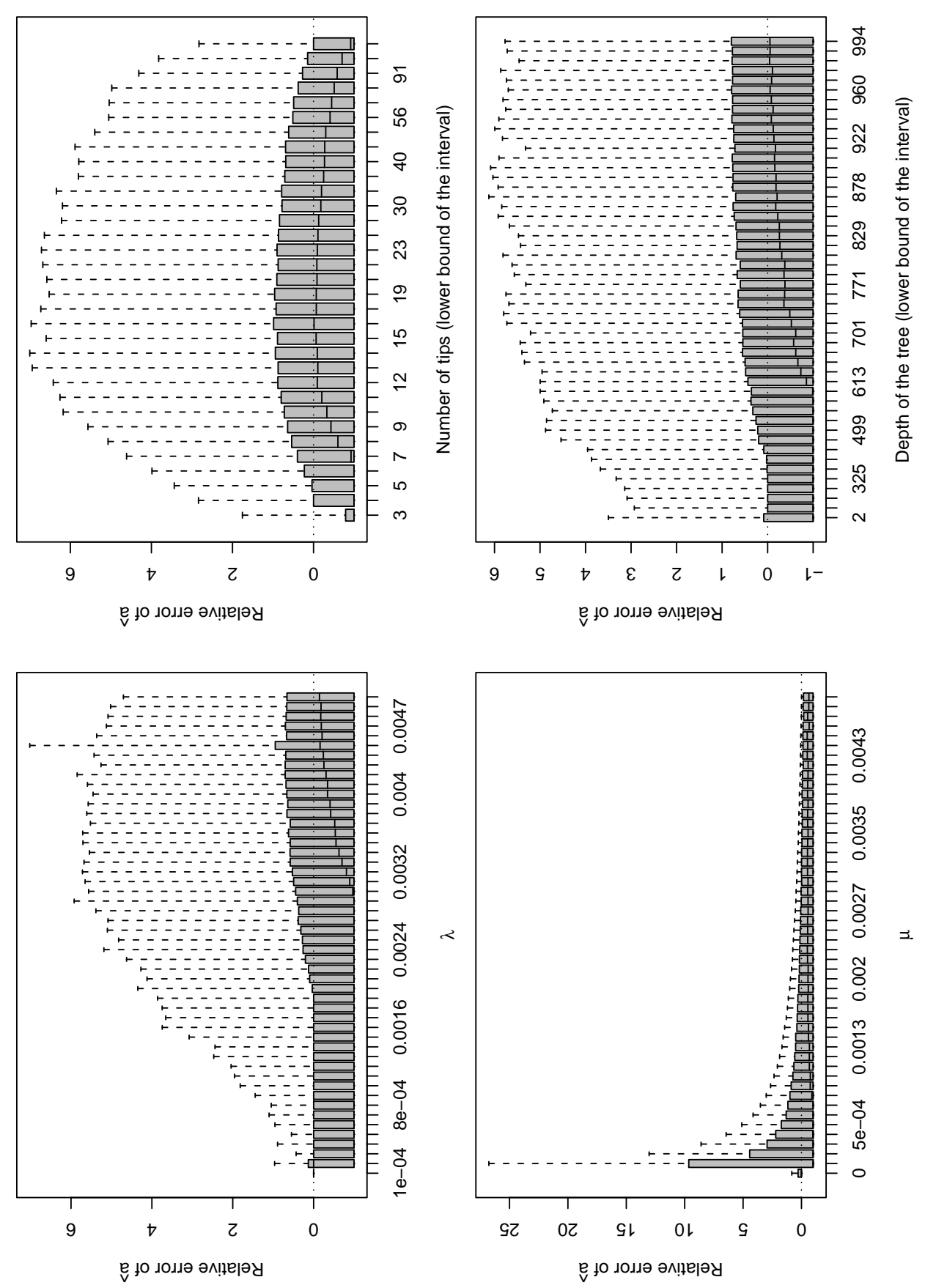

Figure 3: Results for $\hat{a}$. On each plot, the effect is considered alone and the trees are combined for all the different values of the other effects (e.g., the trees with the same value of $\lambda$ may have different number of tips). 

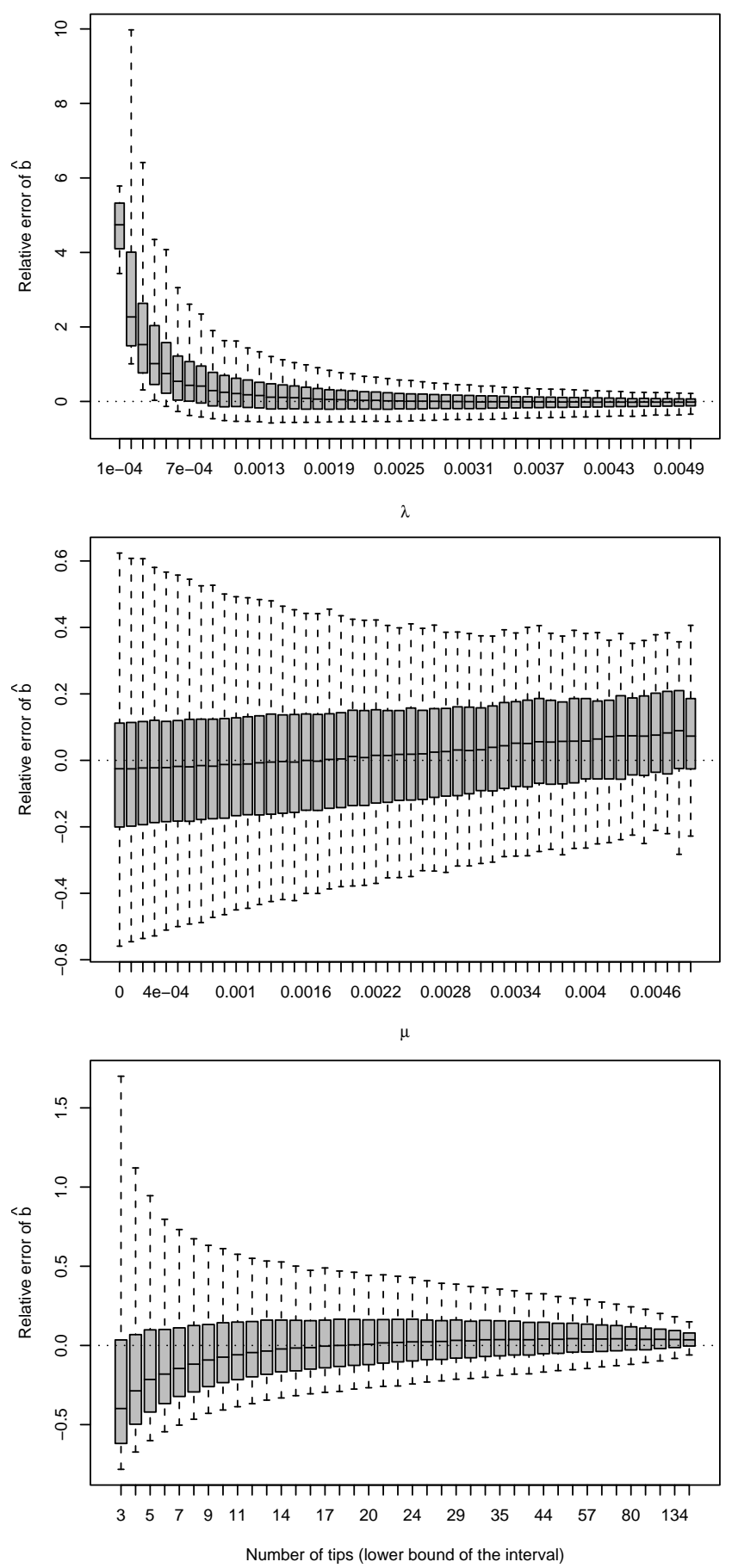

Figure 4: Results for $\hat{b}$. On each plot, the effect is considered alone and the trees are combined for all the different values of the other effects (e.g., the trees with the same value of $\lambda$ may have different number of tips). 

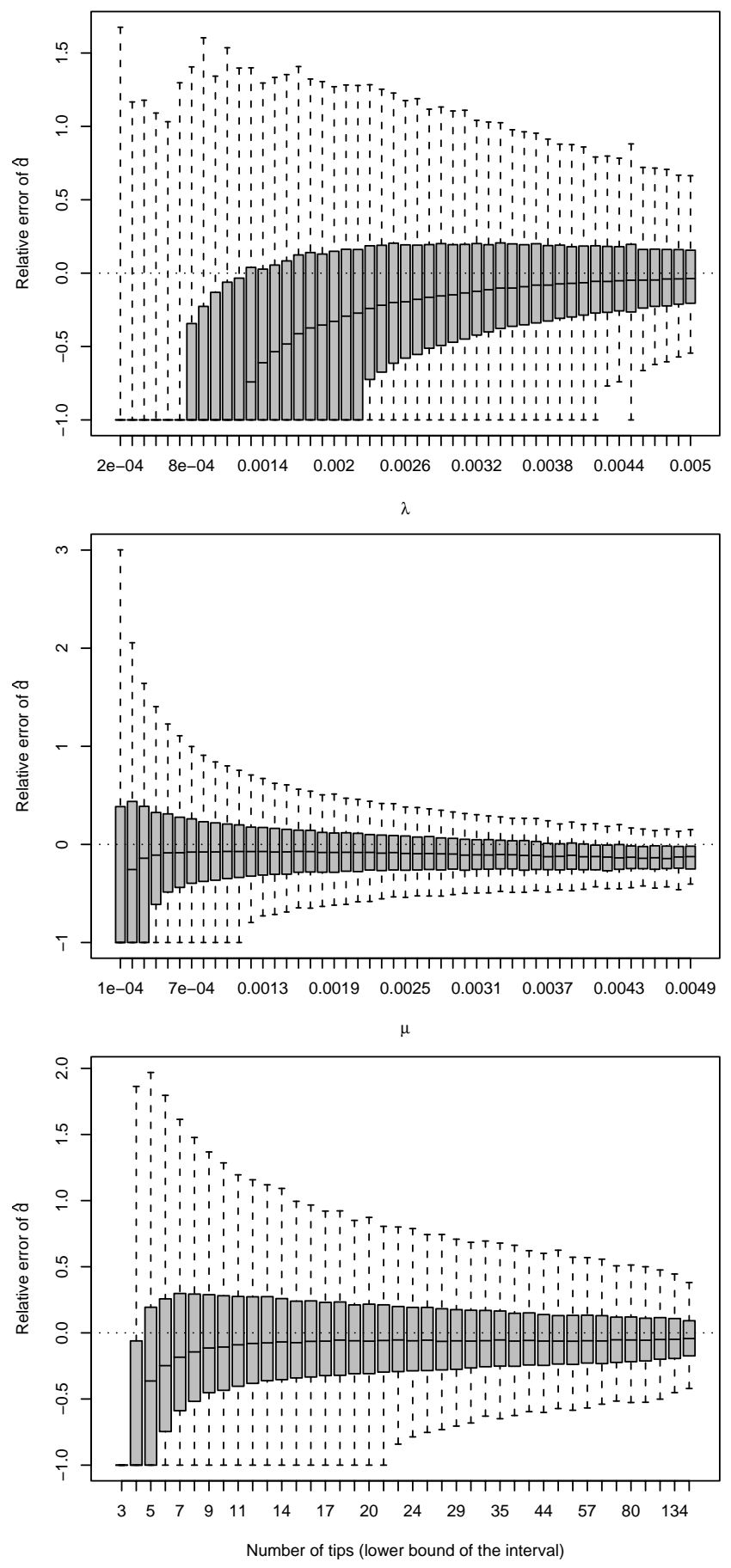

Figure 5: Results for $\hat{d}$. On each plot, the effect is considered alone and the trees are combined for all the different values of the other effects (e.g., the trees with the same value of $\lambda$ may have different number of tips). 

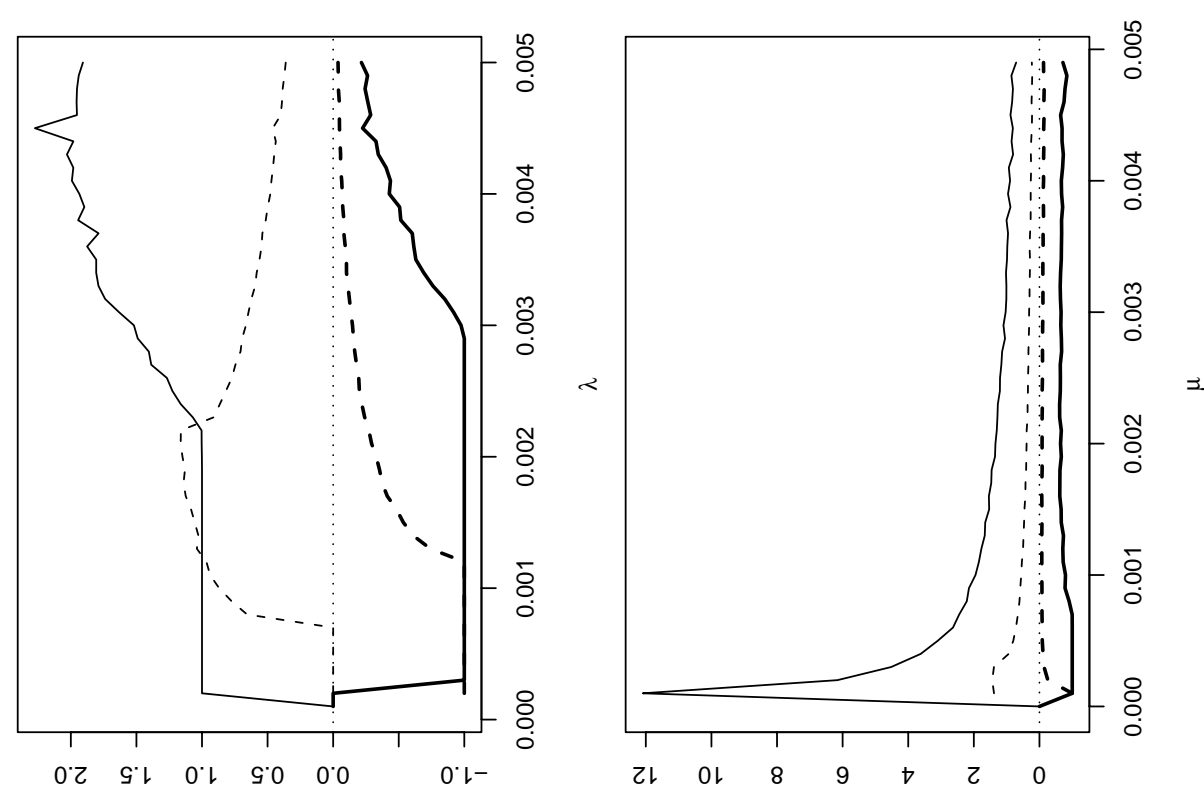

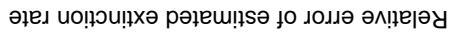

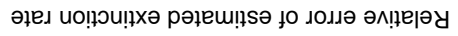
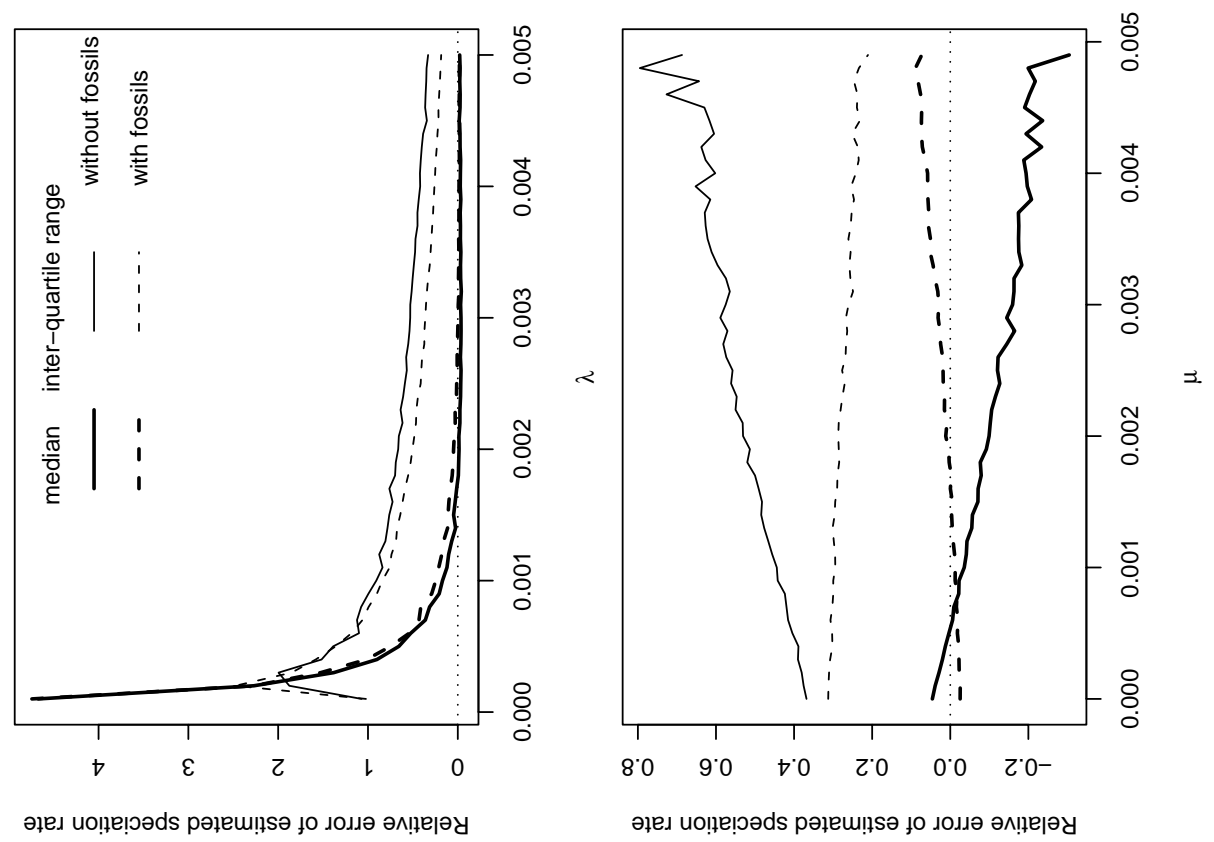

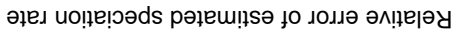

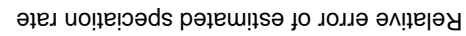

Figure 6: Median and inter-quartile range of relative error of the different estimates of speciation $\left(\hat{b}\right.$ and $\left.\hat{b_{o}}\right)$ and extinction rates $\left(\hat{d}\right.$ and $\left.\hat{d}_{o}\right)$ with respect to the simulated values of speciation $(\lambda)$ or extinction rate $(\mu)$. The inter-quartile range is a measure of the dispersion of the estimates (difference between the first and the third quartiles). 

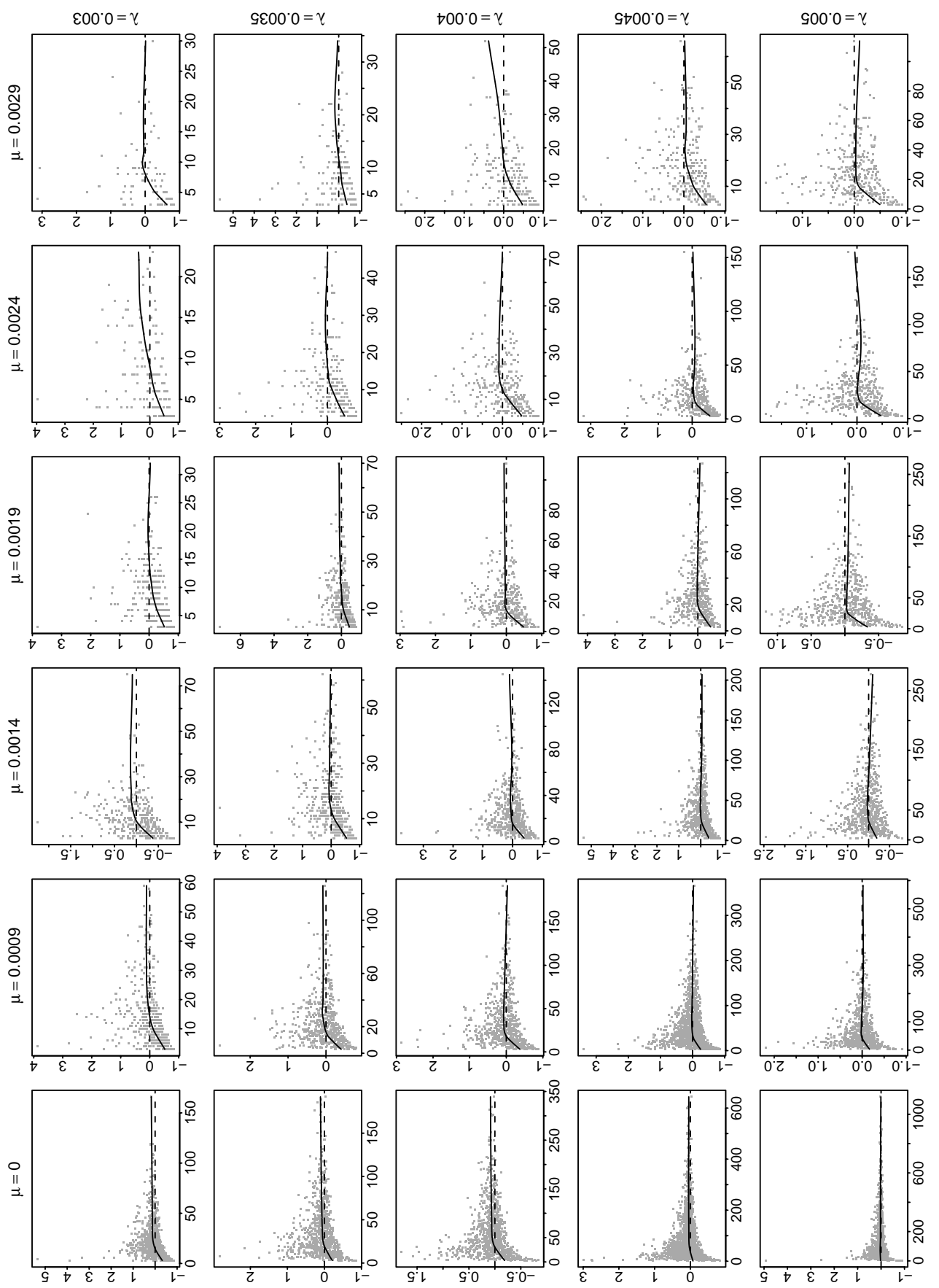

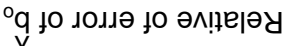

Figure 7: Relative error of the estimates of speciation rate without fossils $\left(\hat{b_{o}}\right)$ with respect to the number of tips of the analysed tree. The parameters $\lambda$ and $\mu$ are the speciation and extinction rates of the simulated trees, and are arranged as rows and columns, respectively. Each grey point is a replication; the lines are local polynomial fits. 

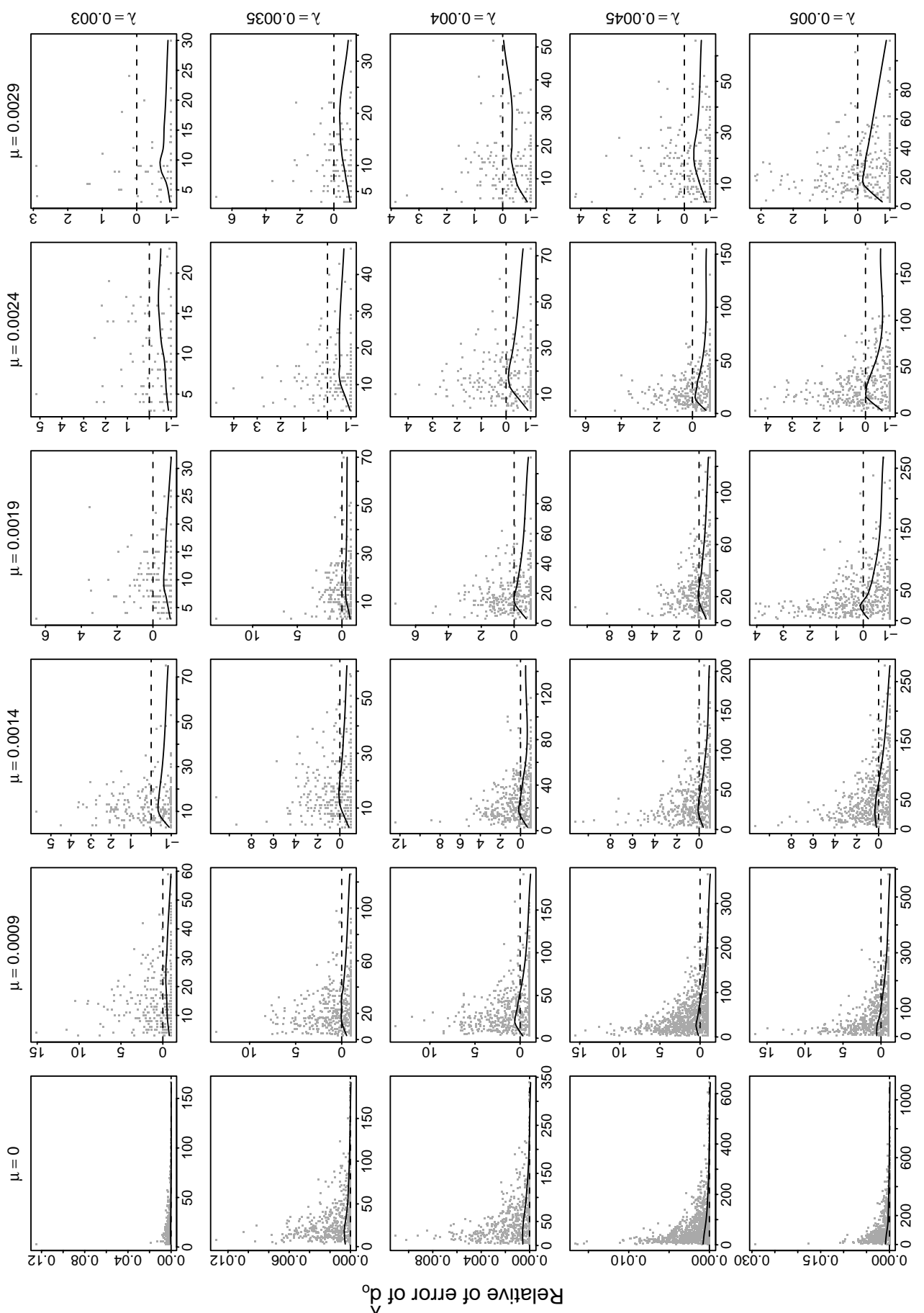

Figure 8: Relative error of the estimates of extinction rate without fossils $\left(\hat{d}_{o}\right)$ with respect to the number of tips of the analysed tree. The parameters $\lambda$ and $\mu$ are the speciation and extinction rates of the simulated trees, and are arranged as rows and columns, respectively. Each grey point is a replication; the lines are local polynomial fits. 